\section{Les suites de points extrémaux liés aux ensembles dans l'espace à 3 dimensions}

\author{
par J. GóRski (Kraków)
}

Introduction. Dans le travail [3] M. F. Lueja a donné la définition d'une suite de points extrémaux liés aux ensembles plans avec quelques applications à la représentation conforme. On peut aisément étendre de ia manière suivante quelques uns de ces résultats au cas de l'espace à 3 pImensions.

Soit $E$ un ensemble borné et fermé dont la capacité $d(E)$ est positive. (Pour la définition de la capacité d'un ensemble voir le travail [1], p. 4.6.) Soit $Q_{1}$ un point quelconque de $E$. Soit le point $Q_{2} \in E$ défini par la formule

$$
1 / Q_{2} Q_{1}=\min _{Q_{E} E}\left(1 / Q Q_{1}\right)
$$

(où $Q_{2} Q_{1}$ est la distance de $Q_{2}$ et $Q_{1}$ ) le point $Q_{3} \in E$ par la condition

$$
\frac{1}{Q_{3} Q_{2}}+\frac{1}{Q_{3} Q_{1}}=\min _{Q_{E} E}\left[\frac{1}{Q Q_{2}}+\frac{1}{Q Q_{1}}\right]
$$

etc. En général, lorsque les points $Q_{1}, Q_{2}, \ldots, Q_{n-1}$ sont définis, soit $Q_{n} \in E$ un point tel que

$$
\sum_{j=1}^{n-1} \frac{1}{Q_{n} Q_{j}}=\min _{Q_{\theta} E} \sum_{j=1}^{n-1} \frac{1}{Q Q_{j}}
$$

On obtient alors une suite infinie $\left\{Q_{n}\right\}$ de points appartenant à $E$

$$
Q_{1}, Q_{2}, Q_{3}, \ldots, Q_{n}, \ldots
$$

Nous l'appellerons suite de points extrémaux liés à l'ensemble $\boldsymbol{E}$.

Potentiel d'équilibre. Désignons par $\sigma_{n}=\sigma_{n}(\Delta)$, où $\Delta$ est un ensemble borelien quelconque, la répartition de la masse unité sur $E$ définie par les points $Q_{1}, Q_{2}, \ldots, Q_{n}$ de la suite (1), c'est-à-dire une fonction d'ensemble $\Delta$ non négative, définie par la formule suivante:

$$
\sigma_{n}(\Delta)=\left\{\begin{array}{l}
0 \quad \text { lorsque } \Delta \text { ne contient aucun des points } Q_{1}, Q_{2}, \ldots, Q_{n}, \\
k / n \quad \text { lorsque } \Delta \text { contient } k \text { des points } Q_{1}, Q_{2}, \ldots, Q_{n} .
\end{array}\right.
$$

D'après le théorème du choix (voir [1]) on peut extraire de la suite $\left\{\sigma_{n}\right\}$ une suite partielle $\left\{\sigma_{n_{k}}\right\}$ convergente vers une fonction d'ensemble $\sigma=\sigma(\Delta)$. Observons qu'on a $\sigma_{n}(E)=\sigma(E)=1$.

On sait [1], p. 33-38, qu'il existe une répartition $\mu$ unique de la masse 1 sur $E$ dite répartition d'équilibre telle qu'on a

(2) $\quad I(\mu)=\int_{i} \int_{i} \frac{d \mu(P) d \mu(Q)}{P Q}=\inf _{\tau \in M} \int_{R} \int_{R} \frac{d \tau(P) d \tau(Q)}{P Q}=\frac{1}{d(E)}$

où $M$ est l'ensemble do toutes les répartitions de la masse 1 sur $E$ (intégrale de Stioltjes-Lebosgue-Radon).

Posons

$$
i_{n}=\sum_{1 \leqslant j<k \leqslant n} \frac{1}{Q_{j} Q_{\eta_{i}}}
$$

D'après la définition des points $Q_{1}, Q_{2}, \ldots, Q_{n}$ on a

$$
s_{n} \leqslant \sum_{j=1}^{n-1} \frac{1}{Q Q_{j}}+\sum_{j=1}^{n-2} \frac{1}{Q Q_{j}}+\ldots+\sum_{j=1}^{2} \frac{1}{Q Q_{j}}+\frac{1}{Q Q_{1}}
$$

pour chaque position du point $Q$ dans $E$.

Partageons l'ensemble $E$ en $s$ ensembles boreliens $\Delta_{1}, \Delta_{2}, \ldots, \Delta_{s}$ et supposons tout d'abord que $Q \in \Delta_{1}$. Multiplions l'inégalité (3) par $\mu\left(\Delta_{1}\right)$. Supposons maintenant que $Q \in \Delta_{2}$ et multiplions (3) par $\mu\left(\Delta_{2}\right)$ etc. Supposons enfin que $Q \epsilon \Delta_{s}$ et multiplions (3) par $\mu\left(\Delta_{s}\right)$. Formons la somme des $s$ inégalités ainsi obtenues. Lorsque $s$ tend vers l'infini et le diamètre de tous les ensembles $\Delta_{1}, \Delta_{2}, \ldots, \Delta_{s}$ tend vers zéro on obtient (en observant que $\left.\mu\left(\Delta_{1}\right)+\ldots+\mu\left(\Delta_{s}\right)=1\right)$ l'inégalité

$$
s_{n} \leqslant \sum_{j=1}^{n-1} \int_{E} \frac{d \mu(Q)}{Q Q_{j}}+\ldots+\sum_{j=1}^{2} \int_{E} \frac{d \mu(Q)}{Q Q_{j}}+\int_{E} \frac{d \mu(Q)}{Q Q_{1}} .
$$

Mais on sait [1], p. 33, que le potentiel d'équilibre

$$
u(P)=\int_{E} \frac{d \mu(Q)}{Q P}
$$

est $\leqslant 1 / d(E)$ sur $E$, il résulte donc de l'inégalité précédente qu'on a

$$
s_{n} \leqslant(n-1) \frac{1}{2} n / d(E) .
$$

D'autre part

$$
s_{n}=\frac{1}{2} \sum_{\substack{i, k=1 \\ j \neq k}}^{n} \frac{1}{Q_{j} Q_{k}}
$$


Soit $N$ un nombre naturel quelconque. Posons

$$
\frac{1}{\left[Q_{j} Q_{k}\right]_{N}}=\left\{\begin{array}{lll}
1 / Q_{j} Q_{k} & \text { si } & 1 / Q_{j} Q_{k} \leqslant N \\
N & \text { si } & 1 / Q_{j} Q_{k}>N .
\end{array}\right.
$$

De (5) et (6) résulte l'inégalité suivante

$$
\frac{2}{n^{2}} s_{n} \geqslant \frac{1}{n^{2}} \sum_{j, k=1}^{n} \frac{1}{\left[Q_{j} Q_{k}\right]_{N}}-\frac{N}{n} .
$$

Le premier terme du second membre peut être représenté par l'intégrale

$$
\int_{E} \int_{E} \frac{1}{[Q P]_{N}} d \sigma_{n}(P) d \sigma_{n}(Q)
$$

De (4) et (7) on déduit l'inégalité

$$
\therefore \frac{n-1}{n} \cdot \frac{1}{d(E)} \geqslant \frac{2}{n^{2}} s_{n} \geqslant \int_{E} \int_{E} \frac{1}{[P Q]_{N}} d \sigma_{n} d \sigma_{n}-\frac{N}{n} .
$$

En maintenant $N$ fixe faison tenđre $n$ vers l'infini par les valeurs $n_{1}, n_{2}, \ldots$ définies plus haut. On obtient l'inégalité

$$
\frac{1}{d(E)} \geqslant \int_{E} \int_{E} \frac{d \sigma d \sigma}{[P Q]_{N}}
$$

et lorsque $N \rightarrow \infty$ on a $I(\mu)=1 / d(E) \geqslant I(\sigma)$.

D'autre part d'après (2) on a $I(\sigma) \geqslant I(\mu)$, donc $I(\sigma)=I(\mu)$ et, puisque la répartition d'équilibre $\mu$ est unique, on a $\sigma \equiv \mu$. Alors la limite de chaque suite partielle convergente extraite de la suite $\left\{\sigma_{n}\right\}$ est égale à $\mu$, d'où il résulte que chaque suite partielle convergente extraite de la suite $u_{n}(P)=\frac{1}{n} \sum_{j=1}^{n} \frac{1}{P Q_{j}}$ est convergente vers la même limite

$$
\int_{R} \frac{d \mu(Q)}{P Q}, \quad P \bar{\epsilon} E
$$

donc la suite $\left\{u_{n}(P)\right\}$ est convergente pour $P \bar{e} E$. Nous obtenons ainsi lo

Thíorìme 1. Le potentiel d'équilibre de l'ensemble $\boldsymbol{E}$ est la limite de la suite

$$
u_{n}(P)=\frac{1}{n} \sum_{j=1}^{n} \frac{1}{P Q_{j}}
$$

quand $P \bar{\epsilon} E$ et $n \rightarrow \infty$, où les points $Q_{j}, j=1,2,3, \ldots$ appartiennent d la suite (1).
Applications au problème de Dirichlet. Soit $f(Q)$ une fonction définie et continue sur $E$ et $Q_{1}^{*}$ un point quelconque de l'ensemble $E$. Soit le point $Q_{2}^{*}$ défini par la formule

$$
\frac{1}{Q_{2}^{*} Q_{1}^{*}}-f\left(Q_{2}^{*}\right)-f\left(Q_{1}^{*}\right)=\min _{Q_{\theta} E}\left[\frac{1}{Q Q_{1}^{*}}-f(Q)-f\left(Q_{1}^{*}\right)\right],
$$

le point $Q_{3}^{*}$ par la condition

$$
\begin{aligned}
\frac{1}{Q_{3}^{*} Q_{2}^{*}}+\frac{1}{Q_{3}^{*} Q_{1}^{*}}-2 f\left(Q_{3}^{*}\right) & -f\left(Q_{2}^{*}\right)-f\left(Q_{1}^{*}\right) \\
& =\min _{Q_{e} E}\left[\frac{1}{Q Q_{2}^{*}}+\frac{1}{Q Q_{1}^{*}}-2 f(Q)-f\left(Q_{2}^{*}\right)-f\left(Q_{1}^{*}\right)\right]
\end{aligned}
$$

etc. En général lorsque les points $Q_{1}^{*}, Q_{2}^{*}, \ldots, Q_{n-1}^{*}$ sont définis, soit $Q_{n}^{*} \epsilon E$ un point tel que

$$
\begin{aligned}
\sum_{j=1}^{n-1} \frac{1}{Q_{n}^{*} Q_{j}^{*}}-(n-1) f\left(Q_{n}^{*}\right) & -\sum_{j=1}^{n-1} f\left(Q_{j}^{*}\right) \\
& =\min _{Q_{A} E}\left[\sum_{j=1}^{n-1} \frac{1}{Q_{Q_{j}^{*}}^{*}}-(n-1) f(Q)-\sum_{j=1}^{n-1} f\left(Q_{j}^{*}\right)\right] .
\end{aligned}
$$

On obtient alors une suite infinie

$$
Q_{1}^{*}, Q_{2}^{*}, Q_{3}^{*}, \ldots, Q_{n}^{*}, \ldots
$$

de points appartenant à $E$ qu'on appelle suite de points extrémaux liés à l'ensemble $E$ et à la fonction $f(Q)$.

Désignons par $\sigma^{*}=\sigma_{n}^{*}(\Delta)$ la répartition de la masse 1 sur $E$ définie par les points $Q_{1}^{*}, Q_{2}^{*}, \ldots, Q_{n}^{*}$ comme à la page 14 . Soit $\sigma^{*}=\sigma^{*}(\Delta)$ la limite d'une suite partielle $\left\{\sigma_{n_{k}}^{*}\right\}$ convergente extraite de la suite $\left\{\sigma_{n}^{*}\right\}$. On sait [2] qu'il existe une répartition unique $\mu^{*}$ de la masse 1 sur $E$ telle qu'on a

$$
I^{*}\left(\mu^{*}\right)=\int_{E} \int_{E} \frac{1}{P Q} d \mu^{*}(P) d \mu^{*}(Q)-2 \int_{E} f(Q) d \mu^{*}(Q)=\inf _{\tau \in M} I^{*}(\tau)
$$

où $M$ est l'ensemble des toutes les répartitions de la masse 1 sur $E$. Posons

$$
s_{n}^{*}=\sum_{1<j<k<n} \frac{1}{Q_{j}^{*} Q_{k}^{*}}-\prod_{k=1}^{(n-1)} \sum_{j=1}^{n} f\left(Q_{j}^{*}\right)
$$

Annoles Polonict Mathematiof 
D'après la définition des points $Q_{1}^{*}, Q_{2}^{*}, \ldots, Q_{n}^{*}$ on a

(10)

$$
\begin{aligned}
\varepsilon_{\eta t}^{*} \leqslant \sum_{j=1}^{n-1} & \frac{1}{Q Q_{j}^{*}}-(n-1) f(Q)-\sum_{j=1}^{n-1} f\left(Q_{j}^{*}\right)+\ldots+ \\
& +\sum_{j=1}^{2} \frac{1}{Q Q_{j}^{*}}-2 f(Q)-\sum_{j=1}^{2} f\left(Q_{j}^{*}\right)+\frac{1}{Q Q_{1}^{*}}-f(Q)-f\left(Q_{1}^{*}\right),
\end{aligned}
$$

pour chaque position du point $Q$ sur $E$. Partageons l'ensemble $E$ en $s$ ensembles boreliens $\Delta_{1}, \Delta_{2}, \ldots, \Delta_{8}$. En procédant de même que à la page 15 on obtient de la formule (10) l'inégalité suivante:

$$
\begin{aligned}
s_{n}^{*} \leqslant & \sum_{j=1}^{n-\ldots 1} \int_{E} \frac{d \mu^{*}(Q)}{Q Q_{j}^{*}}-(n-1) \int_{E} f(Q) d \mu^{*}(Q)-\sum_{j=1}^{n-1} f\left(Q_{j}^{*}\right)+\ldots+ \\
& +\sum_{j=1}^{2} \int_{E} \frac{d \mu^{*}(Q)}{Q Q_{j}^{*}}-2 \int_{E} f(Q) d \mu^{*}(Q)-\sum_{j=1}^{2} f\left(Q_{j}^{*}\right)+ \\
& +\int_{E} \frac{d \mu^{*}(Q)}{Q Q_{1}^{*}}-\int_{E} f(Q) d \mu^{*}(Q)-f\left(Q_{1}^{*}\right) .
\end{aligned}
$$

D'après [2] on a

$$
\text { (12) } \quad u^{*}(P) \stackrel{\mathrm{df}}{=} \int_{E} \frac{d \mu^{*}(Q)}{P Q}-f(P) \leqslant \gamma \quad \text { pour } \quad P \in E_{f},
$$

où $E_{f}$ désigne le noyau de la masse relatif à la distribution $\mu^{*}$. La constante $\gamma$ est égale à

$$
\int_{E} u^{*}(P) d \mu^{*}(P)=1 / d\left(E_{f}\right)-\int_{i} f(P) d \mu_{f}(P),
$$

où $\mu_{f}$ est la distribution d'équilibre pour l'ensemble $E_{f}$.

Supposons maintenant que pour la fonction $f(Q)$ on ait $E_{f}=E$. Il résulte de (11) et (12) qu'on a

(14) $s_{n}^{*} \leqslant(n-1) \gamma-(n-1) \int_{\mathbb{E}} f(Q) d \mu^{*}(Q)+(n-2) \gamma-(n-2) \int_{n} f(Q) d \mu^{*}(Q)+$

$$
\begin{aligned}
& \quad+\ldots+2 \gamma-2 \int_{E} f(Q) d \mu^{*}(Q)+\gamma-\int_{E} f(Q) d \mu^{*}(Q) \\
& =\frac{n(n-1)}{2}\left[\gamma-\int_{E} f(Q) d \mu^{*}(Q)\right] .
\end{aligned}
$$

D'autre part on a

$$
s_{n}^{*}=\frac{1}{2} \sum_{\substack{j, k=1 \\ j \neq k}}^{n} \frac{1}{Q_{j}^{*} Q_{k}^{*}}-\frac{1}{2} \sum_{\substack{j, k=1 \\ j \neq k}}^{n}\left[f\left(Q_{j}^{*}\right)+f\left(Q_{k}^{*}\right)\right]
$$

done (voir page 16)

$$
\frac{2}{n^{2}} s_{n}^{*} \geqslant \frac{1}{n^{2}} \sum_{j, k=1}^{n} \frac{1}{\left[Q_{j}^{*} Q_{k}^{*}\right]_{N}}-\frac{2(n-1)}{n^{2}} \sum_{j=1}^{n} f\left(Q_{j}^{*}\right)-\frac{N}{n} .
$$

Les termes du second membre peuvent être représentés par

$$
\int_{E} \int_{E} \frac{1}{[P Q]_{N}} d \sigma_{n}^{*}(P) d \sigma_{n}^{*}(Q)-2 \frac{n-1}{n} \int_{i} f(Q) d \sigma_{n}^{*}(Q)-\frac{N}{n} .
$$

De (14), (15) et (16) on tire l'inégalité suivante

$$
\begin{aligned}
\frac{n-1}{n}\left[\gamma-\int_{E} f(Q) d \mu^{*}(Q)\right] & \geqslant \frac{2}{n^{2}} s_{n}^{*} \\
& \geqslant \int_{E} \int_{E} \frac{1}{[P Q]_{N}} d \sigma_{n}^{*} d \sigma_{*}^{n}-2 \frac{n-1}{n} \int_{E} f d \sigma_{n}-\frac{N}{n} .
\end{aligned}
$$

En maintenant $N$ fixe faisons tendre $n$ vers l'infini par les valeurs $n_{1}$, $n_{2}, n_{3}, \ldots$ On obtient l'inégalité

$$
\gamma-\int_{E} f(Q) d \mu^{*}(Q) \geqslant \int_{E} \int_{E} \frac{1}{[P Q]_{N}} d \sigma^{*} d \sigma^{*}-2 \int_{E} f(Q) d \sigma^{*}(Q)
$$

et lorsque $N \rightarrow \infty$ on a

$$
\gamma-\int_{j} f(Q) d \mu^{*}(Q) \geqslant I^{*}\left(\sigma^{*}\right)
$$

Mais

$$
\gamma-\int_{E} f(Q) d \mu^{*}(Q)=\int_{E} \int_{E} \frac{1}{P Q} d \mu^{*}(P) d \mu^{*}(Q)-2 \int_{E} f(Q) d \mu^{*}(Q)=I^{*}\left(\mu^{*}\right)
$$

alors $I^{*}\left(\mu^{*}\right) \geqslant I^{*}\left(\sigma^{*}\right)$. Puisque $I^{*}\left(\sigma^{*}\right) \geqslant I^{*}\left(\mu^{*}\right)$, donc $I^{*}\left(\mu^{*}\right)=I^{*}\left(\sigma^{*}\right)$. La distribution remplissant la condition (9) est unique, done $\sigma^{*} \equiv \mu^{*}$. L'ensemble complémentaire de $E$ est une somme finie ou dénombrable de domaines $D_{1}, D_{2}, D_{3}, \ldots$ dont la frontière est contenue dans $\boldsymbol{E}$. Soit $D_{1}$ le domaine contenant le point $\infty$. Les domaines $D_{2}, D_{3}, \ldots$, s'ils existent, sont bornés. 
L'ensemble complémentaire de $E_{f}$ est aussi une somme de domaines $D_{1 f}, D_{2 f}, \ldots$ On sait [2] que la fonction

$$
\int_{\pi} \frac{d \mu^{*}(Q)}{P Q}-\gamma
$$

est la solution du problème de Dirichlet généralisé pour le domaine $D_{i}$, $i=1,2,3, \ldots$ avec la valeur $f(P)$ sur sa frontière (et 0 à l'infini dans le cas $i=1$ ).

Lorsque $E_{f}=E$ on a (voir (13))

$$
\gamma=\frac{1}{d(E)}-\int_{E} f(Q) d \mu(Q)=\frac{1}{d(E)}-\lim _{n \rightarrow \infty} \frac{1}{n} \sum_{j=1}^{n} f\left(Q_{j}\right) .
$$

Nous avons done obtenu le

THÉOR̀̀me 2. Lorsque $E_{f}=E$ la solution du problème de Dirichlet généralisé pour le domaine $D_{\boldsymbol{i}}, i=1,2, \ldots$ avec la valeur $f(P)$ sur sa frontière et 0 à l'infini (dans le cas $i=1$ ) est la limite de la suite

$$
u_{n}^{*}(P)=\frac{1}{n} \sum_{j=1}^{n}\left[\frac{1}{P Q_{j}^{*}}+f\left(Q_{j}\right)\right]-\frac{1}{d(E)}, \quad P \bar{\epsilon} E,
$$

quand $n \rightarrow \infty$, ò̀ les points $Q_{1}^{*}, Q_{2}^{*}, \ldots, Q_{n}^{*}$ appartiennent $\dot{a}$ la suite de points extrémaux (8) et les points $Q_{1}, Q_{2}, \ldots, Q_{n} \dot{a}$ la suite (1).

\section{Travaux cités}

[1] 0. Frostman, Potentiel d'équilibre et capaoité des ensembles avec quelques applications à 2 a théorie des fonetions, Meddel f. Lunds Univ. Mat. Sem. 3 (1935), p. $1-118$

[2] J. Górski, Méthode des points extrémaux de résolution du problème de Divichlet dans l'espace, Ann. Pol. Math. 1 (2) (1955), p. 418-429.

[3] F. Leja, Sur certaines suites liées aux ensembles plans et leur application à la représentation conforme, ce volume, p. 8:13.

Regu par la Rédaction le 1,6.1950

\section{Certains théorèmes concernant la répartition des points extrémaux dans les ensembles plans}

\author{
par J. Gónski et J. SICIAK (Kraków)
}

Introduction. Soit $E$ un ensemble borné et fermé,

$$
E=\sum_{j=1}^{p} E_{j}, \quad E_{j} E_{k}=0 \quad \text { pour } \quad j \neq k,
$$

où $E_{j}, j=1,2, \ldots, p$, est un continu non dégénéré et $f(z)$ une fonction réelle, définie et continue sur $E$. Nous supposons que $E$ est la frontière d'un domaine $D(E)$ contenant le point $z=\infty$. Soit

$$
\omega=|z-\zeta| \exp [-f(z)-f(\zeta)]
$$

et $x^{(n)}=\left\{x_{0}, x_{1}, \ldots, x_{n}\right\}$ un système de $n+1$ points de l'ensemble $\boldsymbol{E}$. Désignons par $V\left(x^{(n)}, \omega\right)$ le produit

$$
V\left(x^{(n)}, \omega\right)=\prod_{0 \leqslant i<k \leqslant n} \omega\left(x_{i}, x_{k}\right)
$$

Un système de $n+1$ points

$$
\eta^{(n)}=\left\{\eta_{0}, \eta_{1}, \ldots, \eta_{n}\right\}
$$

appartenant à $E$ est appelé $n^{\text {-lème }}$ système de points extrémaux de l'ensemble $E$ par rapport à la fonction $\omega$ lorsque

$$
V\left(\eta^{(n)}, \omega\right) \geqslant V\left(x^{(n)}, \omega\right)
$$

pour chaque autre système $x^{(n)}$ de points de l'ensemble $E$.

On sait [3] que, si $\alpha_{n}\left(E_{k}\right)$ désigne le nombre des points extrémaux du $n^{\text {-ième }}$ système qui appartiennent à $E_{k}$, les limites

$$
\lim _{n \rightarrow \infty} \frac{\alpha_{n}\left(E_{k}\right)}{n}=a_{k}(f), \quad k=1,2, \ldots, p
$$

existent. On a

$$
\alpha_{k}(f) \geqslant 0, \quad \sum_{k=1}^{p} \alpha_{k}(f)=1
$$

